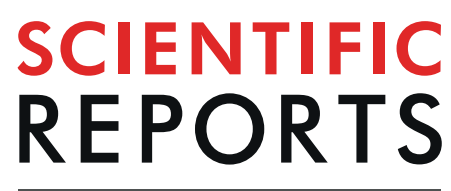

natureresearch

\title{
Molecular serotype-specific identification of Streptococcus pneumoniae using loop-mediated isothermal amplification
}

\author{
Chika Takano ${ }^{1,2,11}$, Yoko Kuramochi ${ }^{3,11}$, Mitsuko Seki ${ }^{1,4^{*}}$, Dong Wook Kim ${ }^{5,6^{*}}$, \\ Daisuke Omagari ${ }^{7}$, Mari Sasano ${ }^{1,8}$, Bin Chang ${ }^{9}$, Makoto Ohnishi ${ }^{9}$, Eun Jin Kim ${ }^{5,6}$, \\ Kazumasa Fuwa ${ }^{2}$, Paul E. Kilgore ${ }^{10}$, Tomonori Hoshino ${ }^{4}$ \& Satoshi Hayakawa ${ }^{1}$
}

In children, the incidence of pneumococcal meningitis has decreased since the introduction of pneumococcal conjugate vaccine (PCV7 and PCV13). However, since the introduction of the vaccine, developed countries have seen the emergence of non-PCV13 serotypes. However, invasive pneumococcal disease (IPD) caused by PCV13-targeted serotypes still represents an important public health problem in resource-limited countries. To develop a rapid, simple, and cost-effective assay to detect serotypes of Streptococcus pneumoniae, we developed a novel loop-mediated isothermal amplification (LAMP) assay based on the sequences available for the 13 capsular types that are included in PCV13: 1, 3, 4, 5, 6A, 6B, 7F, 9V, 14, 18C, 19A, 19F, and $23 \mathrm{~F}$. We evaluated test reactivity, specificity, sensitivity and performance, and compared the results between established LAMP and conventional PCR assays. To support its clinical use, the detection limits of the LAMP assay were evaluated using bacterial genomic DNA-spiked cerebrospinal fluid (CSF) and blood specimens. We confirmed the specificity of the LAMP assay using 41 serotypes of pneumococcal strains. The sensitivity of the LAMP assay was 10 to 100 copies per reaction, compared to 10 to $10^{4}$ copies per reaction for PCR assays. The detection limits of the LAMP assay were comparable when using DNA-spiked CSF and blood specimens, as compared to using purified DNA as the template. In conclusion, a rapid and simple LAMP-based pneumococcal serotyping method has been developed. This is the first report of a LAMP method for a PCV13 serotype-specific identification assay, which could be a promising step to facilitate epidemiological studies of pneumococcal serotyping.

Streptococcus pneumoniae is one of the primary bacterial species responsible for meningitis, bacteraemia, septicaemia, community-acquired pneumonia, and otitis media ${ }^{1}$. Approximately 500,000 children under the age of 5 years die of pneumococcal disease each year, with the majority of these deaths occurring in developing countries $^{2}$. Elderly persons and immunocompromised individuals, including those with HIV/AIDS, sickle cell anaemia, cancer, and end-stage liver or kidney disease, carry the majority of the pneumococcal disease burden in developed countries. Although traditional antimicrobial therapy is effective, pneumococcal resistance to essential antimicrobials, such as penicillin, cephalosporins, and macrolides, is a serious and growing problem worldwide ${ }^{3}$.

${ }^{1}$ Division of Microbiology, Department of Pathology and Microbiology, Nihon University School of Medicine, Tokyo, 173-8610, Japan. ' 2 epartment of Pediatrics and Child Health, Nihon University School of Medicine, Tokyo, 173-8610, Japan. ${ }^{3}$ Nihon University School of Medicine, Tokyo, 173-8610, Japan. ${ }^{4}$ Department of Pediatric Dentistry, School of Dentistry, Meikai University, Saitama, Japan. ${ }^{5}$ Department of Pharmacy, College of Pharmacy, Hanyang University, Ansan, 15588, Republic of Korea. ${ }^{6}$ Institute of Pharmacological Research, Hanyang University, Ansan, 15588, Republic of Korea. ${ }^{7}$ Nihon University School of Dentistry, Tokyo, 101-8310, Japan. ${ }^{8}$ Department of Neurological Surgery, Nihon University School of Medicine, Tokyo, 173-8610, Japan. ${ }^{9}$ Bacteriology I, National Institute of Infectious Diseases, Tokyo, 162-8640, Japan. ${ }^{10}$ Department of Pharmacy Practice, Eugene Applebaum College of Pharmacy \& Health Sciences, Wayne State University, Detroit, MI, 48201, USA. ${ }^{11}$ These authors contributed equally: Chika Takano and Yoko Kuramochi. *email: mitsuko.seki@dent.meikai-u.ac.jp; dongwook@hanyang.ac.kr 
Since the introduction of pneumococcal conjugate vaccine (PCV7 and PCV13), the incidence of meningitis caused by $S$. pneumoniae in children has decreased ${ }^{4,5}$. In developed countries, non-PCV13 serotypes have become an emerging problem since the introduction of the vaccine. However, invasive infections caused by PCV13-targeted serotypes are still a public health problem in resource-limited countries ${ }^{5}$. The disease burden in adults and mortality in adults and children remain high in many countries ${ }^{6}$. In developing countries, the adult disease burden and adult/childhood mortality rates are still high despite international collaboration, such as the Global Alliance for Vaccines and Immunization (GAVI).

Pneumococcus has highly diverse polysaccharide capsule types, containing around 94 different serotypes, and the distribution of serotypes varies geographically ${ }^{7,8}$. The available PCVs were designed to provide immunity against the most prevalent invasive serotypes worldwide ${ }^{8}$. It is crucial to understand the geographical distribution of serotypes and shifts in prevalence over time to optimize vaccine design and assess the impact of vaccine introduction on disease burden.

Conventional bacterial culture methods require a well-equipped laboratory with appropriate biosafety facilities $^{9}$, specialized bacterial culture media, and reagents. Serological typing of pneumococci is performed using the Quellung reaction with type-specific pneumococcal antisera. In this method, serum is added to the bacteria obtained from the medium, and swelling of the capsule is scored under a microscope. Sometimes, the reaction is subtle, and its scoring requires a high degree of technical skill. Well-equipped laboratory facilities and strict control of antisera are also required. Thus, accurately determining pneumococcal serotypes remains challenging due to the limited availability of routine microbiology laboratory services and facilities in developing countries ${ }^{10}$.

End users in developed and developing countries require effective serotype identification tests that meet criteria to ensure global access. Cost-effective, sensitive, and specific diagnostic tests for pneumococcal serotyping are not readily available in many resource-limited countries.

In comparison to loop-mediated isothermal amplification (LAMP), multiplex polymerase chain reaction (PCR)-based assays are relatively expensive and complex to perform in resource-limited laboratory settings because they require a thermal cycler and electrophoretic analysis ${ }^{11-14}$. LAMP is a nucleic acid amplification method that provides rapid, accurate, and cost-effective diagnosis of infectious diseases ${ }^{15,16}$. LAMP-based diagnostic assays for S. pneumoniae, Haemophilus influenzae, and Neisseria meningitidis in cerebrospinal fluid (CSF) specimens have been established ${ }^{17-20}$. LAMP-based methods for meningococcal typing to detect meningococcal serogroups A, B, C, X, Y, and $\mathrm{W}^{21}$, as well as $H$. influenzae serotypes a, b, c, d, e, and f, have been developed ${ }^{17,22}$.

LAMP methods to serotype pneumococcus are not yet available. To develop a rapid, simple, and cost-effective method to detect serotypes of $S$. pneumoniae, we designed LAMP primer sets based on the sequences available for the capsular types 1, 3, 4, 5, 6 A, 6B, 7 F, 9 V, 14, 18 C, 19 A, 19 F, and $23 \mathrm{~F}$ (PCV7 or PCV13 vaccine-targeted serotypes).

\section{Results}

Analytical reactivity and specificity of LAMP-based pneumococcal serotyping. The analytical reactivity and specificity of LAMP-based pneumococcal serotyping (Table 1) were evaluated using 55 pneumococcal strains belonging to 41 pneumococcal serotypes (Table 2). A genomic DNA concentration of $10^{5}$ copies per reaction was used as a standard for each strain. The LAMP primer sets for capsular types 1, 3, 4, 5, 6B, 7 F, 9 V, $14,18 \mathrm{C}, 19 \mathrm{~A}, 19 \mathrm{~F}$, and $23 \mathrm{~F}$ successfully amplified the target DNA sequence of each target locus (Table 1). LAMP primer sets for capsular types $1,3,4,5$, and 14, which are single-serotype serogroups, did not have any DNAamplified product from DNA of other target capsular types.

The LAMP primer set for capsular type 6B also amplified DNA of capsular types 6 A, 6C, and 6D. DNA of other capsular types was not amplified. Capsular types $6 \mathrm{~A}, 6 \mathrm{~B}, 6 \mathrm{C}$, and $6 \mathrm{D}$ share almost the same sequence of putative rhamnosyl transferase gene $(w c i P)^{23}$. An important point mutation in this sequence is related to serotype switching, e.g. capsular type $6 \mathrm{~B}$ changes to $6 \mathrm{~A}^{24}$. To detect capsular types $6 \mathrm{~A}, 6 \mathrm{~B}, 6 \mathrm{C}$, and $6 \mathrm{D}$, we designed the backward inner primer (BIP) corresponding to the middle of the $\mathrm{B} 1$ region where the important mutation is found (Table 1).

The LAMP primer set for capsular type $7 \mathrm{~F}$ amplified DNA of capsular type $7 \mathrm{~A}$. Capsular types $7 \mathrm{~A}$ and $7 \mathrm{~F}$ share the same sequence of serotype7A/7F-specific oligosaccharide repeat unit polymerase $(w z y)^{25}$. DNA of other capsular types, including capsular type $7 \mathrm{C}$, was not amplified. Bentley et al. reported that the $w z y$ sequences of capsular types $7 \mathrm{~B}$ and $7 \mathrm{C}$ differ from those of capsular types $7 \mathrm{~A}$ and $7 \mathrm{~F}^{25}$. The polymerization linkage of $w z y$ in types $7 \mathrm{~B}$ and $7 \mathrm{C}$ is $\mathrm{D}-\mathrm{Glcp}(\beta 1-4) \mathrm{D}-\mathrm{Glcp}$; in types $7 \mathrm{~A}$ and $7 \mathrm{~F}$, it is $\mathrm{D}-\mathrm{Glcp}(\beta 1-3) \mathrm{D}$-GalpNAc.

The LAMP primer set for capsular type $9 \mathrm{~V}$ amplified DNA of capsular type $9 \mathrm{~A}$. Capsular types $9 \mathrm{~A}$ and $9 \mathrm{~V}$ share the same sequence of serotype $9 \mathrm{~A} / 9 \mathrm{~V}$-specific $w z y$ gene. DNA of other target capsular types, including the capsular type $9 \mathrm{~N}$, was not amplified. Capsular type $9 \mathrm{~N}$ has a different $w z y$ sequence ${ }^{25}$. The polymerization linkage of $w z y$ in types $9 \mathrm{~A}$ and $9 \mathrm{~V}$ is D-Glcp $(\beta 1-4) \mathrm{D}$-Glcp, and D-Glcp $(\beta 1-4) \mathrm{D}-\mathrm{GlcpNAc}$ in type $9 \mathrm{~N}^{25}$.

The LAMP primer set for capsular type $18 \mathrm{C}$ amplified DNA of capsular types $18 \mathrm{~A}, 18 \mathrm{~B}$, and $18 \mathrm{~F}$. Capsular types $18 \mathrm{~A}, 18 \mathrm{~B}, 18 \mathrm{C}$, and $18 \mathrm{~F}$ share the same sequence of the serotype $18 \mathrm{~A} / 18 \mathrm{~B} / 18 \mathrm{C} / 18 \mathrm{~F}$-specific wzy gene ${ }^{25}$. DNA of other capsular types was not amplified.

The LAMP primer set for capsular type 19 A targeting the wzy gene for serotype 19 A detected capsular type 19 A only. DNA of other capsular types, including $19 \mathrm{~F}$, was not detected by this primer set. Likewise, the LAMP primer set for capsular type $19 \mathrm{~F}$ that targeted the $w z y$ gene for serotype $19 \mathrm{~F}$ detected capsular type $19 \mathrm{~F}$ only. DNA of other target capsular types, including $19 \mathrm{~A}$, was not detected by this primer set. The wzy genes for $19 \mathrm{~A}$ and $19 \mathrm{~F}$ have different sequences ${ }^{25}$.

Pimenta et al. reported difficulty in differentiating between capsular types $19 \mathrm{~A}$ and $19 \mathrm{~F}$ when they used PCR-based pneumococcal serotyping methods ${ }^{13}$. We designed a LAMP primer set corresponding to the specific region of each capsular type after alignment analysis of $w z y$ sequences for the two capsular types. The LAMP primer set consisted of six primers including eight regions of the target sequences, while the PCR primer sets 


\begin{tabular}{|c|c|c|c|}
\hline $\begin{array}{l}\text { Serotyping } \\
\text { primer name }\end{array}$ & LAMP Primer Sequence (Sequence $5^{\prime}-3^{\prime}$; Reaction temperature, $63^{\circ} \mathrm{C}$ ) & \begin{tabular}{|l|}
$\begin{array}{l}\text { Length (base } \\
\text { pairs) }\end{array}$ \\
\end{tabular} & $\begin{array}{l}\text { Gene/GenBank no./target } \\
\text { serotypes }\end{array}$ \\
\hline 4_F3 & CAT TCA GAA GTA CAA AAT TAT CAG GA & 26 & \multirow{6}{*}{$w z y /$ CR931635/4 } \\
\hline 4_B3 & ACG CTT TAT AAC TCG GGA C & 19 & \\
\hline 4_FIP & $\begin{array}{l}\text { GCT CTA ACT GCT AGT ACT GTT TTA GAT TGT ACA ATG CGG } \\
\text { GTA GG }\end{array}$ & 44 & \\
\hline 4_BIP & TTC AGC ATA TTC AGA GGC AGC CAA GGA GAA CAC ACC AGG & 39 & \\
\hline 4_LF & ATT ACC CTA GAA ATA AAG CCC ACT C & 25 & \\
\hline 4_LB & TCA GGA ATA ATA GAT GAT TTA GGA GT & 26 & \\
\hline 6B_F3 & GGG ATT GAA TTA CCG AAC AT & 20 & \multirow{6}{*}{$\begin{array}{l}w c i \text { P/CR931639/6A, 6B, } \\
\text { 6C, 6D }\end{array}$} \\
\hline 6B_B3 & GTC CAT GTC TTC GAT ACA AGA & 21 & \\
\hline 6B_FIP & $\begin{array}{l}\text { GGA ACC ATC TCT AGC AAT GCA TGA TTA GTA TTT TAT TCA } \\
\text { TGC CTA TAT CTG G }\end{array}$ & 52 & \\
\hline 6B_BIP & $\begin{array}{l}\text { CTG TCT CAT GAT A } \underline{A}^{\mathrm{a}} \text { T TAT TTT GCA AAG AGT TGC TCA GGG } \\
\text { CAG AAC }\end{array}$ & 45 & \\
\hline 6B_LF & AAA CCT GCA GTA CAC CC & 17 & \\
\hline 6B_LB & TTT GCA CTA GAG TAT GGG AAG & 21 & \\
\hline 9V_F3 & AGC GAT TCG TAT TTT TGA AGA & 21 & \multirow{6}{*}{$w z y / C R 931648 / 9 \mathrm{~V}, 9 \mathrm{~A}$} \\
\hline 9V_B3 & TCA ACA TTG TCA GTA GCG T & 19 & \\
\hline 9V_FIP & $\begin{array}{l}\text { CGG AGT TAA CGA TAA TCC CAT TTG TAA TTT TTG GTT TGG } \\
\text { AAA AGG AC }\end{array}$ & 47 & \\
\hline 9V_BIP & $\begin{array}{l}\text { TAC TAG ATA TAC TTG CTC GAA CGG GTT CCA AGA AAT AGA } \\
\text { CTT AGA AGA AC }\end{array}$ & 50 & \\
\hline 9V_LF & CCA AGC ATT GAA ATC AAT A & 19 & \\
\hline 9V_LB & CGG GTA TTT TAT TTG TAG TG & 20 & \\
\hline 14_F3 & GAG GAA TCC CTA AAA GCT AT & 20 & \multirow{6}{*}{ wzy/CR931662/14 } \\
\hline 14_B3 & CAA AAT ACT GAC AAA GCT AGA & 21 & \\
\hline 14_FIP & $\begin{array}{l}\text { GTG ACC CCA ATA A } \underline{A}^{\mathrm{b}} \text { A TAT CTA CTG TAG GGA ATG GAA ATG } \\
\text { TTA CTT GGC G }\end{array}$ & 49 & \\
\hline 14_BIP & $\begin{array}{l}\text { AGG ACA GGA GTT TTA GGA AGT ATA ATA AGT CTC TCA GAT } \\
\text { GAA TCA CA }\end{array}$ & 47 & \\
\hline 14_LF & AGG GAA TTC TGA CAC CTG & 18 & \\
\hline 14_LB & CAG TAA TGT TTT ATT ATC TG & 20 & \\
\hline 18C_F3 & ATT CGA TGG CTA GAA CAG AT & 20 & \multirow{6}{*}{$\begin{array}{l}\text { wzy/CR931673/18 (18A, } \\
18 \mathrm{~B}, 18 \mathrm{C}, 18 \mathrm{~F})\end{array}$} \\
\hline 18C_B3 & AGC ATT TCT ATA AAG AAG AGT GT & 23 & \\
\hline 18C_FIP & $\begin{array}{l}\text { TGT TAC AAA CCC TAT CCC TCT CCA AGG GAG TTG AAT CAA } \\
\text { CCT A }\end{array}$ & 43 & \\
\hline 18C_BIP & $\begin{array}{l}\text { ATG GTC TTA CAG GGA CAA TGG GTC CTA CAA ATC CTA TCT } \\
\text { CAA TGT }\end{array}$ & 45 & \\
\hline 18C_LF & CCA TAA ATA TAG GGG CGA & 18 & \\
\hline 18C_LB & GAT CCA TAA TGA TAT TTT GAA GTA C & 25 & \\
\hline 19F_F3 & TGG ATT TGT TGG TTT AAT AGC AG & 23 & \multirow{6}{*}{$w z y / \mathrm{CR} 931678 / 19 \mathrm{~F}$} \\
\hline 19F_B3 & GAT AAT TAA CTA GGC CCA TTT CC & 23 & \\
\hline 19F_FIP & $\begin{array}{l}\text { CAC TCT CAA ATA GCG TCC TAG TCG GTA TTC CAG CAT TTT } \\
\text { ACT ACT CTT }\end{array}$ & 48 & \\
\hline 19F_BIP & $\begin{array}{l}\text { GAG GCT CAA TTC AGC ATT TTA ATC AGG CAC CAA TGT TTC } \\
\text { ACT G }\end{array}$ & 43 & \\
\hline 19F_LF & TGA ACG ACC GGC TAA AAA CA & 20 & \\
\hline 19F_LB & AGA TCC TGG TGA AGT TTT TGG & 21 & \\
\hline 23F_F3 & GGC GTT AAC ATT TTT TTT CAA AC & 23 & \multirow{6}{*}{$w z y / \mathrm{CR} 931685 / 23 \mathrm{~F}$} \\
\hline 23F_B3 & CAA CTA ACC CAA CAT AAC CAT & 21 & \\
\hline 23F_FIP & $\begin{array}{l}\text { GCA TCC CCA AAA AAC AAA TGA AAC CAA TCA TAT AGC CAT } \\
\text { CGA GTG }\end{array}$ & 45 & \\
\hline 23F_BIP & $\begin{array}{l}\text { CCT TTG GAA ATA CGA CGA AGG GGT AAA GGC ATC TCT ACC } \\
\text { GTT }\end{array}$ & 42 & \\
\hline 23F_LF & AAA AAA ATT CAC AAC ACC T & 19 & \\
\hline 23F_LB & TGG ACA CAA TAT TAG AAG TG & 20 & \\
\hline 1_F3 & CAG CTA GTC GTA ATT TAC AGA T & 22 & \multirow{6}{*}{ wzy/CR931632/1 } \\
\hline 1_B3 & TTA CAA TTC CAA AGT ATC CTC C & 22 & \\
\hline 1_FIP & $\begin{array}{l}\text { CCA AGT TTG ATT AGA ATA CCC CGT GCA ATT ATT TCG AAG } \\
\text { GTC GT }\end{array}$ & 44 & \\
\hline 1_BIP & $\begin{array}{l}\text { TGG ACA CCT TTT TTC CAA ACG TTT CAC ATA TCC CTC TCC } \\
\text { CAC }\end{array}$ & 42 & \\
\hline 1_LF & CTT ACT ATG TCA TTA AAA AAA G & 22 & \\
\hline 1_LB & TCA ACC AAA TAT GGT TTT ACT CT & 23 & \\
\hline \multicolumn{4}{|l|}{ Continued } \\
\hline
\end{tabular}




\begin{tabular}{|c|c|c|c|}
\hline $\begin{array}{l}\text { Serotyping } \\
\text { primer name }\end{array}$ & LAMP Primer Sequence (Sequence $5^{\prime}-3^{\prime}$; Reaction temperature, $63^{\circ} \mathrm{C}$ ) & \begin{tabular}{|l|}
$\begin{array}{l}\text { Length (base } \\
\text { pairs) }\end{array}$ \\
\end{tabular} & $\begin{array}{l}\text { Gene/GenBank no./target } \\
\text { serotypes }\end{array}$ \\
\hline 3_F3 & GCC TGT TAG ATA TGA AGA TGT TTC & 24 & \multirow{6}{*}{ galU/CR931634/3 } \\
\hline 3_B3 & ATG TAT CAA TAG CAT CTG TCA AT & 23 & \\
\hline 3_FIP & $\begin{array}{l}\text { TTG GTT TCT CTA CAA AAG CAT CAA CTT CTT ATG GTG TGA } \\
\text { TTT CTC CT }\end{array}$ & 47 & \\
\hline 3_BIP & $\begin{array}{l}\text { TTG GAC GTT ATC TAC TTA CTC CTG ATT CAT TAC CTG CTC } \\
\text { CTG G }\end{array}$ & 43 & \\
\hline 3_LF & AGA GGC CAT TAC TAC TTT CCA ATC & 24 & \\
\hline 3_LB & GAT TTT TTC TAT ATT AGA AAC CCA & 24 & \\
\hline 5_F3 & CCC ATG ATT TAT GCC CTC T & 19 & \multirow{6}{*}{ wzy/CR931637/5 } \\
\hline 5_B3 & TGT TTC AGA ATG TTC ACC AAC & 21 & \\
\hline 5_FIP & $\begin{array}{l}\text { GGC ATT GAC AGT ATA AGA AAA AGC ACA ACG TTC TTC TTC } \\
\text { TCA TCG T }\end{array}$ & 46 & \\
\hline 5_BIP & TTG AAG GTT ACG CGC CAT TTG TGT ATT CAG AAG GCA ACC & 39 & \\
\hline 5_LF & GGG CTA AAA AAA GCA TGC GGA & 21 & \\
\hline 5_LB & GGT GCC AAG AGT TTT ATT CTT TGG & 24 & \\
\hline 7F_F3 & ATT ATT TGG CTA TTC AAC AGG A & 22 & \multirow{6}{*}{$w z y / \mathrm{CR} 931643 / 7 \mathrm{~F}, 7 \mathrm{~A}$} \\
\hline 7F_B3 & GAA CAA TCC TAT AAA TCC ATT CTC A & 25 & \\
\hline 7F_FIP & $\begin{array}{l}\text { AGT CTG CCA AAC ATC TCC ATA AAA CTA GTT CTG ATT TTG } \\
\text { GTC GG }\end{array}$ & 44 & \\
\hline 7F_BIP & $\begin{array}{l}\text { AGA GGC GGA AAT TTC AAA AAT TCC GTG AAC AGA TAG TAA } \\
\text { TGG GTG TA }\end{array}$ & 47 & \\
\hline 7F_LF & GAG ATT ATT TGA ACA ATT GAA CT & 23 & \\
\hline 7F_LB & GAT ATT TAG TGG TTC C & 16 & \\
\hline 19A_F3 & AGC TCT TAC TAT TAT AGT TGA CCT & 24 & \multirow{5}{*}{ wzy/CR931675/19A } \\
\hline 19A_B3 & GAG CGT TTA TGA CTA TAA ATG AAG A & 25 & \\
\hline 19A_FIP & $\begin{array}{l}\text { GAA CCA CTG AAA ATT TGA ACC CGT TAG GAG AGA GAT TCA } \\
\text { TAA TCT TGC }\end{array}$ & 48 & \\
\hline 19A_BIP & $\begin{array}{l}\text { TAC CAG TTA TGA AGG TGA GCT AAC ATC CAA AAA TAT AAG } \\
\text { CAG ATA CGT }\end{array}$ & 48 & \\
\hline 19A_LB & GTG CGA ACT TCG ATT CGG G & 19 & \\
\hline
\end{tabular}

Table 1. LAMP primer sequences in this study. ${ }^{a}$ point mutation related to serotype switching. ${ }^{b}$ original sequence was $\mathrm{T}$ (to avoid formation of primer dimer and nonspecific reactions).

consisted of only two primers (forward and reverse primers) including two regions of the target sequences. Therefore, in comparison to the PCR primers, the LAMP primer set should easily distinguish between those two capsular types.

The LAMP primer set targeting the $w z y$ gene for capsular type $23 \mathrm{~F}$ detected capsular type $23 \mathrm{~F}$ only. DNA of other capsular types, including capsular type $23 \mathrm{~A}$, was not detected, as the $w z y$ genes for $23 \mathrm{~A}$ and $23 \mathrm{~F}$ have different sequences ${ }^{25}$.

LAMP-amplified products were analysed by direct DNA sequencing to confirm the specificity. The obtained sequences were compared with those of the target region of the original sequence at each capsulation locus (between F1 and B1; Supplementary Fig. S1), and identity to the expected nucleotide sequences was confirmed (Fig. S2).

Detection limit of LAMP-based pneumococcal serotyping method. The detection limits of the LAMP assay were 10 genome copies per reaction for capsular types $14,18 \mathrm{C}, 19 \mathrm{~F}$, and $23 \mathrm{~F}$; and $10^{2}$ genome copies per reaction for capsular types $1,3,4,5,6 \mathrm{~B}, 7 \mathrm{~F}, 9 \mathrm{~V}$, and $19 \mathrm{~A}$. The detection limits of the PCR assay were 10 genome copies per reaction for capsular type $19 \mathrm{~F} ; 10^{2}$ genome copies per reaction for capsular types $4,6 \mathrm{~B}, 7 \mathrm{~F}$, $9 \mathrm{~V}, 14,18 \mathrm{C}, 19 \mathrm{~A}$, and $23 \mathrm{~F} ; 10^{3}$ genome copies per reaction for capsular types 1 and 3 ; and $10^{4}$ genome copies per reaction for capsular type 5 (Table 3 ). For six of the serotypes, the sensitivity of the LAMP assay was 10- to 100 -fold greater than that of PCR-based pneumococcal serotyping. The products were visually inspected by monitoring the turbidity/colour of the reaction tube and utilizing real-time turbidimetry and a real-time colorimetric sensor (Fig. 1). The detection limits of each serotyping LAMP assay were identical in the real-time measurement and direct visual inspection. No LAMP amplification was detected in control samples lacking target DNA. The experiments were repeated in triplicate over 3 days, and identical results were obtained in laboratories both in Japan and South Korea.

LAMP analysis of DNA-spiked specimens. The LAMP assay detected 10-100 genome copies of the genomic DNA even when using DNA-spiked CSF specimens as the template. The results were identical to those obtained with purified DNA as the template (Table 3). The detection limits of PCR-based pneumococcal serotyping assay for serotypes $6 \mathrm{~B}, 14$, and $23 \mathrm{~F}$ decreased from $10^{2}$ to $10^{3}$ genome copies per reaction. The detection limits for other serotypes were the same as when purified DNA was used as the template. 
Using DNA-spiked blood specimens, the detection limits of the LAMP assay were slightly attenuated from 10 copies to 100 copies for serotypes $18 \mathrm{C}$ and $23 \mathrm{~F}$; other serotypes were detected with sensitivity as high as with purified DNA as the template. In contrast, the detection limits of all PCR-based pneumococcal serotyping assay changed from $10-10^{3}$ to $10^{3}-10^{5}$ or more genome copies per reaction (Table 3 ). Again, the results of the LAMP assay were identical between direct visual inspection and real-time measurement.

LAMP assays using colorimetric visual inspection dye plus a real-time colorimetric sensor. The results of LAMP assay using colorimetric visual inspection dye and a real-time colorimetric sensor are shown in Fig. 1. The colour change of the LAMP reagents was readily observed. Detection time of the LAMP reaction decreased from 19 minutes to 8 minutes when the colorimetric dye was used with pyrophosphatase (Isothermal Master Mix, no dye; Canon Medical Systems Corporation, Tochigi, Japan). This reaction protocol can increase the LAMP reaction speed.

\section{Discussion}

In children under 5 years old, the incidence of non-vaccine serotype-specific invasive pneumococcal diseases has increased worldwide because of the widespread introduction of PCV7 or PCV13 ${ }^{5,26}$. In adults, the number of clinical infections with such non-vaccine pneumococcal-serotype-specific strains has also increased ${ }^{6}$. In Japan, PCV usage has reduced antibiotic resistance ${ }^{27}$, while the most common serotypes of pneumococcal strains are still PCV7 or PCV13 vaccine-targeted strains. At the same time, diseases due to $S$. pneumoniae that were not included in the vaccines (such as serotypes $8,10 \mathrm{~A}, 12 \mathrm{~F}, 15 \mathrm{~A}, 23 \mathrm{~A}$, and $24 \mathrm{~F}$ ) have been reported ${ }^{5,28}$.

To optimize vaccine design and assess the impact on disease burden following vaccine introduction, it is crucial to understand the geographic distribution of serotypes and shifts in prevalence over time. Although such studies are typically conducted in central laboratories with equipment and qualified personnel, the future application of nucleic acid detection methods such as LAMP have potentially important roles in diagnosing a number of prevalent pathogens, particularly in low-resource countries. S. pneumoniae is one of these pathogens. The use of standard pneumococcal serotyping assays is limited in developing countries, and the accurate determination of pneumococcal serotyping remains a challenge ${ }^{10}$. For these reasons, future studies are anticipated to compare serotyping classification and accuracy using traditional methods with LAMP methods. The Quellung reaction using type-specific pneumococcal antisera and a microscope, the conventional serological typing of pneumococcus, requires microbiology laboratory services, expensive antisera and experienced technician.

Varying degrees of success have been achieved using multiplex PCR-based assays for pneumococcal serotyping. However, in contrast to LAMP assays, PCR requires thermal cyclers, electrophoresis, UV lamps and technicians with expertise in using equipment to run PCR assays.

The pneumococcal serotype-specific LAMP assay showed good performance in differentiating each target pneumococcal capsular type. The pneumococcal serotype-specific LAMP assay was analytically specific and had a better detection limit compared to conventional pneumococcal serotype-specific PCR. The high sensitivity of this pneumococcal serotype-specific LAMP assay is consistent with previous studies ${ }^{17-19,21}$.

To conduct a pilot evaluation of the pneumococcal serotyping LAMP assay, we used two methods for DNA preparation. CSF specimens were simply heated and centrifuged. A commercially available kit (Procedure for Ultra Rapid Extraction, PURE; Eiken Chemical) was used for the blood. PURE can produce a DNA solution suitable for the LAMP reaction within 10 minutes without the use of a centrifuge. It can be used with dried blood spots, which can be extended to field study ${ }^{29}$.

The pneumococcal serotype-specific LAMP reaction demonstrated equivalent sensitivity with spiked CSF samples and purified DNA template. LAMP reactions were not inhibited, or were inhibited only slightly, when using DNA-spiked CSF and blood. PCR is inhibited by biological substances, particularly heparin ${ }^{30}$ and other blood components, including haem, leukocyte DNA, and immunoglobulin $\mathrm{G}^{22,31,32}$. The LAMP assay can be performed using simple DNA preparation methods because the LAMP reaction more readily tolerates potentially disturbing biological elements (i.e. reaction inhibitors) than $\mathrm{PCR}^{33}$.

The LAMP method requires only the preparation of a reaction mixture and placement of the tube into some incubator at $63^{\circ} \mathrm{C}$. Amplicon of the target DNA can be detected easily by visual inspection, with no requirement for specialized equipment to read the results. Furthermore, due to its high sensitivity and robustness of the reaction, DNA sample preparation can be simplified, such as by boiling or using the PURE method.

The robustness, superior detection limit and simple performance of the LAMP assay make it an excellent alternative to pneumococcal serotype-specific $\mathrm{PCR}^{34}$. Although the detection accuracy should be further improved, the convenience of the LAMP assay could facilitate surveillance of pneumococcal serotypes compared to PCR.

This study assessed pneumococcal serotype-specific LAMP products using a reaction mixture including pyrophosphatase. In the reaction, rather than a "white precipitate", we observed a colour change from colourless to violet due to triphenylmethane dye, which binds to double-stranded DNA (D-QUICK; Kaneka Co., Osaka, Japan), indicating a positive reaction ${ }^{35}$. To determine the LAMP amplification results, we usually observe a white precipitate of magnesium pyrophosphate, which is the by-product of LAMP reaction. As an alternative, we observed a colour change of the reaction mixture using a thermostatic colour sensor (MyAbscope ${ }^{\circledR}$; Kaneka Co., Osaka, Japan) that measures the absorbance of the reaction mixture in real time. As shown in Fig. 1, the detection time was reduced when pyrophosphatase was used in the reaction mixture, compared to using conventional LAMP reagents without pyrophosphatase. Pyrophosphate, a by-product of the LAMP reaction, attenuates the activity of DNA polymerase, and addition of pyrophosphatase can increase the LAMP reaction speed.

This is the first report of a PCV13 pneumococcal serotype-specific identification assay using the LAMP method. Using this method, decreases in PCV13 serotypes associated with vaccine use can easily be observed. Development of the serotype-specific LAMP assay represents a promising step to facilitate epidemiological studies of pneumococcal serotyping. Based on this study, further LAMP-based methods are currently under 


\begin{tabular}{|c|c|c|c|c|c|c|c|c|c|c|c|c|c|c|c|c|}
\hline \multirow[b]{2}{*}{ Vaccine } & \multirow[b]{2}{*}{ Serotypes } & \multirow{2}{*}{$\begin{array}{l}\text { No. of } \\
\text { strains }\end{array}$} & \multirow[b]{2}{*}{ Strain ID } & \multirow{2}{*}{$\begin{array}{l}\text { Origin } \\
\text { of } \\
\text { isolate }\end{array}$} & \multicolumn{12}{|c|}{ LAMP primer set } \\
\hline & & & & & 4 & $6 B$ & $9 \mathrm{~V}$ & 14 & $18 \mathrm{C}$ & $19 \mathrm{~F}$ & $23 \mathrm{~F}$ & 1 & 3 & 5 & $7 F$ & 19A \\
\hline \multirow{7}{*}{ PCV7 } & 4 & 2 & SP0852, SP0143 & $\mathrm{RT}^{\mathrm{a}}$, CSF & $+^{\mathrm{b}}$ & - & - & - & - & - & - & - & - & - & - & - \\
\hline & $6 \mathrm{~B}$ & 2 & SP0857, SP1489 & $\mathrm{N}, \mathrm{N}$ & - & + & - & - & - & - & - & - & - & - & - & - \\
\hline & $9 \mathrm{~V}$ & 2 & SP0916, SP2928 & $\mathrm{N}, \mathrm{B}$ & - & - & + & - & - & - & - & - & - & - & - & - \\
\hline & 14 & 2 & SP0869, SP3320 & $\mathrm{N}, \mathrm{B}$ & - & - & - & + & - & - & - & - & - & - & - & - \\
\hline & $18 \mathrm{C}$ & 2 & SP0873, SP2818 & $\mathrm{N}, \mathrm{B}$ & - & - & - & - & + & - & - & - & - & - & - & - \\
\hline & $19 \mathrm{~F}$ & 2 & SP0862, SP1118 & $\mathrm{N}, \mathrm{N}$ & - & - & - & - & - & + & - & - & - & - & - & - \\
\hline & $23 \mathrm{~F}$ & 2 & SP0885, SP2838 & $\mathrm{N}, \mathrm{N}$ & - & - & - & - & - & - & + & - & - & - & - & - \\
\hline \multirow{6}{*}{ PCV13 } & 1 & 2 & SP3121, SP3070 & B, B & - & - & - & - & - & - & - & + & - & - & - & - \\
\hline & 3 & 2 & SP1441, SP3256 & $\mathrm{N}, \mathrm{N}$ & - & - & - & - & - & - & - & - & + & - & - & - \\
\hline & 5 & 2 & SP3034, ATCC6305 & $\mathrm{U}, \mathrm{U}$ & - & - & - & - & - & - & - & - & - & + & - & - \\
\hline & $6 \mathrm{~A}$ & 2 & SP1567, SP1589 & $\mathrm{N}, \mathrm{N}$ & - & + & - & - & - & - & - & - & - & - & - & - \\
\hline & $7 \mathrm{~F}$ & 2 & SP3365, SP3172 & B, RT & - & - & - & - & - & - & - & - & - & - & + & - \\
\hline & $19 \mathrm{~A}$ & 2 & SP1516, SP3081 & $\mathrm{N}, \mathrm{N}$ & - & - & - & - & - & - & - & - & - & - & - & + \\
\hline \multirow{11}{*}{ PPSV23 } & 2 & 1 & D39 & $\mathrm{U}$ & - & - & - & - & - & - & - & - & - & - & - & - \\
\hline & 8 & 1 & ATCC6308 & $\mathrm{U}$ & - & - & - & - & - & - & - & - & - & - & - & - \\
\hline & $9 \mathrm{~N}$ & 1 & SP2700 & CSF & - & - & - & - & - & - & - & - & - & - & - & - \\
\hline & $10 \mathrm{~A}$ & 1 & SP1933 & $\mathrm{N}$ & - & - & - & - & - & - & - & - & - & - & - & - \\
\hline & $11 \mathrm{~A} / \mathrm{E}$ & 1 & SP2760 & RT & - & - & - & - & - & - & - & - & - & - & - & - \\
\hline & $12 \mathrm{~F}$ & 1 & SP0113 & B & - & - & - & - & - & - & - & - & - & - & - & - \\
\hline & $15 \mathrm{~B}$ & 1 & SP3354 & $\mathrm{N}$ & - & - & - & - & - & - & - & - & - & - & - & - \\
\hline & $17 \mathrm{~F}$ & 1 & NCTC11904 & $\mathrm{U}$ & - & - & - & - & - & - & - & - & - & - & - & - \\
\hline & 20 & 1 & SP2830 & $\mathrm{N}$ & - & - & - & - & - & - & - & - & - & - & - & - \\
\hline & $22 \mathrm{~F}$ & 1 & SP1854 & $\mathrm{N}$ & - & - & - & - & - & - & - & - & - & - & - & - \\
\hline & $33 \mathrm{~F}$ & 1 & SP3201 & $\mathrm{N}$ & - & - & - & - & - & - & - & - & - & - & - & - \\
\hline \multirow{17}{*}{$\begin{array}{l}\text { Non- } \\
\text { vaccine } \\
\text { serotypes }\end{array}$} & $6 \mathrm{C}$ & 1 & SP3362 & $\mathrm{N}$ & - & + & - & - & - & - & - & - & - & - & - & - \\
\hline & $6 \mathrm{D}$ & 1 & SP2739 & $\mathrm{N}$ & - & + & - & - & - & - & - & - & - & - & - & - \\
\hline & 7A & 1 & $2040 / 37$ & $\mathrm{U}$ & - & - & - & - & - & - & - & - & - & - & + & - \\
\hline & 7C & 1 & SP3285 & $\mathrm{N}$ & - & - & - & - & - & - & - & - & - & - & - & - \\
\hline & 9A & 1 & Wilder & $\mathrm{U}$ & - & - & + & - & - & - & - & - & - & - & - & - \\
\hline & 13 & 1 & SP0073 & $\mathrm{N}$ & - & - & - & - & - & - & - & - & - & - & - & - \\
\hline & $15 \mathrm{~A}$ & 1 & SP2758 & \begin{tabular}{|l|} 
RT \\
\end{tabular} & - & - & - & - & - & - & - & - & - & - & - & - \\
\hline & $15 \mathrm{C}$ & 1 & SP3343 & $\mathrm{N}$ & - & - & - & - & - & - & - & - & - & - & - & - \\
\hline & $18 \mathrm{~A}$ & 2 & SP0852, 8609/43 & B, U & - & - & - & - & + & - & - & - & - & - & - & - \\
\hline & $18 \mathrm{~B}$ & 1 & SP1901 & $\mathrm{N}$ & - & - & - & - & + & - & - & - & - & - & - & - \\
\hline & $18 \mathrm{~F}$ & 1 & Lederle & $\mathrm{U}$ & - & - & - & - & + & - & - & - & - & - & - & - \\
\hline & $23 \mathrm{~A}$ & 1 & SP3374 & $\mathrm{N}$ & - & - & - & - & - & - & - & - & - & - & - & - \\
\hline & $24 \mathrm{~F}$ & 1 & SP3193 & $\mathrm{N}$ & - & - & - & - & - & - & - & - & - & - & - & - \\
\hline & 34 & 1 & SP3359 & $\mathrm{N}$ & - & - & - & - & - & - & - & - & - & - & - & - \\
\hline & $35 \mathrm{~B}$ & 1 & SP3357 & $\mathrm{N}$ & - & - & - & - & - & - & - & - & - & - & - & - \\
\hline & 37 & 1 & SP2742 & $\mathrm{N}$ & - & - & - & - & - & - & - & - & - & - & - & - \\
\hline & 38 & 1 & SP3356 & $\mathrm{N}$ & - & - & - & - & - & - & - & - & - & - & - & - \\
\hline
\end{tabular}

Table 2. Reactivity and specificity of the pneumococcal serotyping LAMP assay. ${ }^{\mathrm{a}} \mathrm{RT}$, respiratory tract specimen; CSF, cerebrospinal fluid; N, nasopharyngeal swab; B, blood; U, unknown. ${ }^{\text {b }}+$, Amplification within 25 min incubation; -, no amplification within 120 min incubation.

preparation for PPSV23 targets and other serotypes predicted to emerge after the widespread use of PCV13. This study represents the first step toward achieving our ultimate objective of developing LAMP-based methods covering all pneumococcal serotypes.

\section{Materials and Methods}

Bacterial strains. In this study, we analysed 55 strains of $S$. pneumoniae, including serotypes that belong to PCV7, PCV13, and PPSV23, and a number of non-vaccine serotypes. The PCV7 serotypes were serotype 4 (SP0852, SP0143), serotype 6B (SP0857, SP1489), serotype 9V (SP0916, SP2928), serotype 14 (SP0869, SP3320), serotype 18 C (SP0873, SP2818), serotype 19F (SP0862, SP1118), and serotype 23 F (SP0885, SP2838). Additional PCV13 serotypes were serotype 1 (SP3121, SP3070), serotype 3 (SP1441, SP3256), serotype 5 (SP3034, ATCC6305), serotype 6 A (SP1567, SP1589), serotype 7 F (SP3365, SP3172), and serotype 19 A (SP1516, SP3081). PPSV23 serotypes were serotype 2 (D39), serotype 8 (ATCC6308), serotype 9 N (SP2700), serotype 10 A 


\begin{tabular}{|l|l|l|l|l|l|l|}
\hline \multirow{2}{*}{$\begin{array}{l}\text { S.pneumoniae } \\
\text { serotypes }\end{array}$} & \multicolumn{2}{|l|}{\begin{tabular}{l} 
Detection limit (Purified DNA) \\
\cline { 2 - 7 }
\end{tabular}} & $\begin{array}{l}\text { Detection limit (DNA } \\
\text { spiked CSF }\end{array}$ & \multicolumn{2}{l|}{$\begin{array}{l}\text { Detection limit (DNA } \\
\text { spiked blood) }\end{array}$} \\
\hline 4 & PCR $^{\mathbf{b}, \mathbf{c}}$ & LAMP & PCR & LAMP & PCR & LAMP \\
\hline $6 \mathrm{~B}$ & $10^{2}$ copies $^{\mathrm{d}}$ & $10^{2}$ & $10^{2}$ & $10^{2}$ & $>10^{5}$ & $10^{2}$ \\
\hline $9 \mathrm{~V}$ & $10^{2}$ & $10^{2}$ & $10^{3}$ & $10^{2}$ & $10^{5}$ & $10^{2}$ \\
\hline 14 & $10^{2}$ & $10^{2}$ & $10^{2}$ & $10^{2}$ & $>10^{5}$ & $10^{2}$ \\
\hline $18 \mathrm{C}$ & $10^{2}$ & 10 & $10^{3}$ & 10 & $10^{5}$ & 10 \\
\hline $19 \mathrm{~F}$ & 10 & 10 & $10^{2}$ & 10 & $>10^{5}$ & $10^{2}$ \\
\hline $23 \mathrm{~F}$ & $10^{2}$ & 10 & 10 & 10 & $10^{3}$ & 10 \\
\hline 1 & $10^{3}$ & 10 & $10^{3}$ & 10 & $>10^{5}$ & $10^{2}$ \\
\hline 3 & $10^{3}$ & $10^{2}$ & $10^{3}$ & $10^{2}$ & $>10^{5}$ & $10^{2}$ \\
\hline 5 & $10^{4}$ & $10^{2}$ & $10^{3}$ & $10^{2}$ & $10^{5}$ & $10^{2}$ \\
\hline $7 \mathrm{~F}$ & $10^{2}$ & $10^{2}$ & $10^{4}$ & $10^{2}$ & $>10^{5}$ & $10^{2}$ \\
\hline $19 \mathrm{~A}$ & $10^{2}$ & $10^{2}$ & $10^{2}$ & $10^{2}$ & $>10^{5}$ & $10^{2}$ \\
\hline
\end{tabular}

Table 3. Detection limits of LAMP and PCR assays detecting DNAs of pneumococcal serotypes and using the DNA spiked CSF specimens. ${ }^{a}$ Cerebrospinal fluid specimen collected between 1998 and $2002^{39}$. ${ }^{b} \mathrm{PCR}$ results were obtained by electrophoretic analysis. LAMP results were determined visually. ${ }^{c}$ conventional PCR (serotypes $1,3,4,5,6 \mathrm{~B}, 18 \mathrm{C}, 19 \mathrm{~F}$ and $23 \mathrm{~F}^{11}$; serotypes $9 \mathrm{~V}, 7 \mathrm{~F}^{12}$; serotype $14^{14}$; serotype $19 \mathrm{~A}^{13}$ ). ${ }^{\mathrm{d}}$ Number of genome copies per reaction.

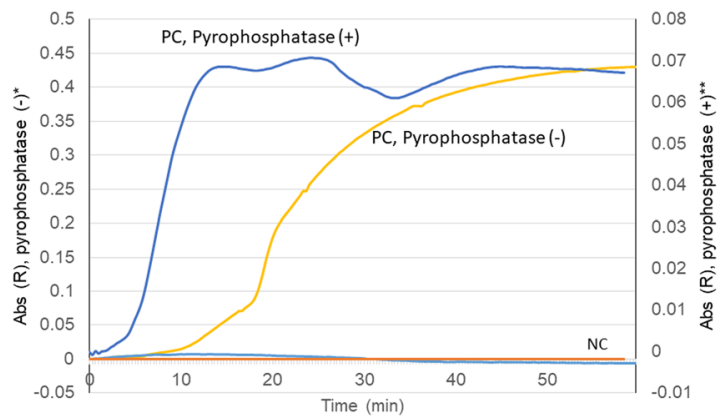

Figure 1. The relationship between reaction time and the absorbance of the reaction tubes. Colorimetric dye can be used with pyrophosphatase. Pyrophosphate, a by-product of the LAMP reaction, attenuates the activity of DNA polymerase. The addition of pyrophosphatase can increase the LAMP reaction speed. *, Bst DNA Polymerase (New England Biolabs, Ipswich, MS, USA) \& conventional LAMP reagent; **, Isothermal Master Mix (no dye; Canon Medical Systems Corporation, Tochigi, Japan); PC, positive control; NC, negative control.

(SP1933), serotype 11 A/E (SP2760), serotype 12 F (SP0113), serotype 15B (SP3354), serotype 17 F (NCTC11904), serotype 20 (SP2830), serotype $22 \mathrm{~F}$ (SP1854), and serotype $33 \mathrm{~F}$ (SP3201). Non-vaccine serotypes were serotype 6C (SP3362), serotype 6D (SP2739), serotype 7 A (2040/37), serotype 7 C (SP3285), serotype 9 A (Wilder), serotype 13 (SP0073), serotype 15 A (SP2758), serotype 15 C (SP3343), serotype 18 A (SP0852, 8609/43), serotype 18B (SP1901), serotype 18 F (Lederle), serotype 23 A (SP3374), serotype 24F (SP3193), serotype 34 (SP3359), serotype 35B (SP3357), serotype 37 (SP2742), and serotype 38 (SP3356) strains. By Quellung reaction using type-specific pneumococcal antisera (Statens Serum Institute, Copenhagen, Denmark), the capsule production of the 54 reference strains was identified in advance. Strain SP2760 was indicated as serotype $11 \mathrm{~A} / \mathrm{E}$ because serotypes $11 \mathrm{~A}$ and $11 \mathrm{E}$ could not be discriminated by the Quellung reaction.

Preparation of chromosomal DNA. Chromosomal DNA from the 55 strains was prepared using a Wizard ${ }^{\circledR}$ Genomic DNA Purification Kit (Promega, Fitchburg, WI, USA) according to the manufacturer's recommendations. The concentration of chromosomal DNA was measured with a NanoDrop 1000 spectrophotometer (Thermo Fisher Scientific Inc., Waltham, MA, USA). The genome copy number was estimated based on the molecular size of S. pneumoniae strain R6 (2.0 Mbp; GenBank accession number, NC_003098). Each DNA sample was diluted to $10^{5} \mathrm{DNA}$ copies/reaction and used to evaluate the specificity of assays. For the detection limit study, serial tenfold dilutions of genomic DNA from PCV13 serotypes (capsular types 1, 3, 4, 5, 6B, $7 \mathrm{~F}$, 9V, 14, 18 C, 19 A, 19 F, and 23 F, which are SP0852, SP0857, SP0916, SP0869, SP0862, SP0885, SP3121, SP1441, SP3034, SP1567, SP3365, and SP1516, respectively) were amplified by LAMP, and we then compared the results with those of conventional PCR ${ }^{11-14}$. To determine the detection limit, triplicate LAMP testing of S. pneumoniae was carried out using serial tenfold dilutions of chromosomal DNA over a 3-day period. The supernatant of pooled S. pneumoniae-negative CSF specimens was used in the spiking assay ${ }^{36}$. Tenfold dilutions of each serotype of S. pneumoniae genomic DNA were amplified using the established LAMP and conventional PCR assays ${ }^{11-14}$. 
LAMP primer design. As shown in Table 1, 12 LAMP primer sets for $S$. pneumoniae were designed with reference to the published sequences in GenBank and using LAMP primer design software ${ }^{37}$. The LAMP primer set for S. pneumoniae consisted of two outer primers (F3 and B3), a forward inner primer (FIP), a backward inner primer (BIP), and loop primers (LF and/or LB).

LAMP reaction. The LAMP procedure used in this study was described previously ${ }^{22}$. Briefly, we carried out LAMP in a reaction mixture consisting of $1.6 \mu \mathrm{M}$ each of FIP and BIP, $0.2 \mu \mathrm{M}$ each of F3 and B3, $0.4 \mu \mathrm{M}$ of LF/ LB, 8 U of $B s t$ DNA polymerase large fragment (New England Biolabs, Ipswich, MA, USA), 1.4 mM deoxynucleoside triphosphates, $0.8 \mathrm{M}$ betaine (Sigma, St. Louis, MO, USA), $20 \mathrm{mM}$ Tris- $\mathrm{HCl}$ (pH 8.8), $10 \mathrm{mM} \mathrm{KCl}, 10 \mathrm{mM}$ $\left(\mathrm{NH}_{4}\right)_{2} \mathrm{SO}_{4}, 8 \mathrm{mM} \mathrm{MgSO}_{4}, 0.1 \%$ Tween 20 , and template DNA, and the final volume was adjusted to $25 \mu \mathrm{L}$ with distilled water. We incubated each reaction mixture at $63^{\circ} \mathrm{C}$ for 60 minutes and then heated at $80^{\circ} \mathrm{C}$ for 2 minutes for termination of the reaction.

Analysis of LAMP products. The turbidity of the reaction tube was determined in real-time by reading the optical density at $650 \mathrm{~nm}\left(\mathrm{OD}_{650}\right)$ at 6-s intervals using a Loopamp ${ }^{\circledR}$ real-time turbidimeter (LA-500; Eiken Chemical Co., Tokyo, Japan). We calculated the amplification time required to exceed a turbidity of 0.1 ( Tt $)$ using the turbidimeter software, as described previously ${ }^{38}$. The detection limit was measured using a colorimetric visual inspection dye (leucotriphenylmethane ${ }^{35}$; D-QUICK, Kaneka Co., Osaka, Japan), Isothermal Master Mix (no dye; Canon Medical Systems Corporation, Tochigi, Japan), and a thermostatic colour sensor (MyAbscope ${ }^{\circledR}$; Kaneka Co., Osaka, Japan). The colour change in the reaction mixture was examined at intervals of $20 \mathrm{~s}$. Amplification time was determined with the thermostatic colour sensor software, as when the $\mathrm{OD}_{650}$ exceeded 0.01 .

Each amplified LAMP product was sequenced at Akita Prefectural University Biotechnology Centre using a BigDye $^{\circledR}$ Terminator V3.1 cycle sequencing kit (Applied Biosystems, Foster City, CA, USA) and a 3130xL Genetic Analyser (Applied Biosystems), and their sequences were verified using the primers shown in the Supplementary Table.

PCR assay. PCR was carried out in $25-\mu \mathrm{L}$ reaction mixtures containing $1 \mathrm{U}$ Ex Taq DNA polymerase (TaKaRa Bio, Tokyo, Japan), $0.2 \mathrm{mM}$ of each deoxyribonucleoside triphosphate, $10 \mathrm{mM}$ Tris-HCl buffer (pH 8.3), $50 \mathrm{mM}$ $\mathrm{KCl}, 2 \mathrm{mM} \mathrm{MgCl}, 0.5 \mu \mathrm{M}$ of each primer, and $2 \mu \mathrm{L}$ of template DNA. Amplification was carried out with two thermal cyclers: Veriti ${ }^{\mathrm{TM}}$ (Applied Biosystems, Foster City, CA, USA) and T-100 ${ }^{\mathrm{TM}}$ (Bio-Rad, Hercules, CA, USA) for 35 cycles of denaturation at $95^{\circ} \mathrm{C}$ for $30 \mathrm{~s}$, primer annealing at $54^{\circ} \mathrm{C}$ for $90 \mathrm{~s}$, and extension at $72^{\circ} \mathrm{C}$ for $60 \mathrm{~s}$, with a final incubation at $72^{\circ} \mathrm{C}$ for 10 minutes ${ }^{11-14}$. The PCR products were electrophoresed on agarose gels and visualized by staining with ethidium bromide.

DNA-spiked clinical CSF and blood specimens. As a pilot evaluation of the pneumococcal serotyping LAMP assays, 39 pneumococcal PCR-negative specimens were randomly chosen from CSF collected in a previous bacterial meningitis study in Hanoi, Vietnam ${ }^{36}$. The specimens of CSF were incubated at $95^{\circ} \mathrm{C}$ for 2 minutes as pre-treatment and centrifuged at $13,000 \times g$ for 5 minutes. The supernatants of these specimens were kept for use in DNA-spiked CSF experiments.

Blood was collected from five healthy volunteers. Blood samples were heparinized for storage. Using Procedure for Ultra Rapid Extraction (PURE; Eiken Chemical, Tokyo), DNA from the blood samples was prepared and used for DNA-spiked blood experiments.

Ethical declaration. We used CSF specimens preserved from our previous surveillance study ${ }^{36,39}$. All CSF specimens used in this study were de-identified prior to laboratory processing and analysis. Ethical approval for patient specimen collection during surveillance was obtained from the following ethics review committees: The Institutional Review Board (IRB) of the International Vaccine Institute, Seoul, South Korea, and the IRB at the National Institute of Hygiene and Epidemiology, Hanoi, Vietnam. Each institution participated in prospective, population-based surveillance of childhood meningitis from 1999 to $2002^{36,39}$. During these surveillance studies, written consent was not obtained as CSF collection was considered routine standard care for hospitalized children with suspected bacterial meningitis. Therefore, verbal consent from the parent or legal guardian present with the child during the period of hospitalization was recorded in the patient's medical chart at the time of the clinical lumbar puncture procedure. This consent procedure was approved by the local scientific ethics review committees of the participating institutions.

We received informed consent prior to collecting blood from five healthy volunteers. The procedures were approved by the IRB of Nihon University School of Medicine (IRB No. 28-9-0/1). All experiments were performed in accordance with relevant guidelines and regulations.

Received: 18 June 2019; Accepted: 9 December 2019;

Published online: 27 December 2019

\section{References}

1. Brown, P. D. \& Lerner, S. A. Community-acquired pneumonia. Lancet 352, 1295-1302 (1998).

2. O'Brien, K. L. et al. Burden of disease caused by Streptococcus pneumoniae in children younger than 5 years: global estimates. Lancet 374, 893-902 (2009).

3. Jacoby, G. A. Antimicrobial-resistant pathogens in the 1990s. Annu Rev Med 47, 169-179 (1996).

4. Martin, N. G., Sadarangani, M., Pollard, A. J. \& Goldacre, M. J. Hospital admission rates for meningitis and septicaemia caused by Haemophilus influenzae, Neisseria meningitidis, and Streptococcus pneumoniae in children in England over five decades: a population-based observational study. Lancet Infect Dis 14, 397-405 (2014). 
5. Waight, P. A. et al. Effect of the 13-valent pneumococcal conjugate vaccine on invasive pneumococcal disease in England and Wales 4 years after its introduction: an observational cohort study. Lancet Infect Dis 15, 535-543 (2015).

6. McGill, F., Heyderman, R. S., Panagiotou, S., Tunkel, A. R. \& Solomon, T. Acute bacterial meningitis in adults. Lancet 388, 3036-3047 (2016).

7. Messaoudi, M. et al. The relevance of a novel quantitative assay to detect up to 40 major Streptococcus pneumoniae serotypes directly in clinical nasopharyngeal and blood specimens. PLoS One 11, e0151428 (2016).

8. Johnson, H. L. et al. Systematic evaluation of serotypes causing invasive pneumococcal disease among children under five: the pneumococcal global serotype project. PLoS Med 7, e1000348 (2010).

9. World Health Organization, G. Laboratory biosafety manual - third edition. http://www.who.int/csr/resources/publications/ biosafety/WHO_CDS_CSR_LYO_2004_11/en/(2004).

10. Seki, M. et al. Loop-mediated isothermal amplification methods for diagnosis of bacterial meningitis. Front Pediatr 6, 57 (2018).

11. Pai, R., Gertz, R. E. \& Beall, B. Sequential multiplex PCR approach for determining capsular serotypes of Streptococcus pneumoniae isolates. J Clin Microbiol 44, 124-131 (2006).

12. da Gloria Carvalho, M. et al. Revisiting pneumococcal carriage by use of broth enrichment and PCR techniques for enhanced detection of carriage and serotypes. J Clin Microbiol 48, 1611-1618 (2010).

13. Pimenta, F. C. et al. Rarely occurring 19A-like cps locus from a serotype $19 \mathrm{~F}$ pneumococcal isolate indicates continued need of serology-based quality control for PCR-based serotype determinations. J Clin Microbiol 47, 2353-2354 (2009).

14. Dias, C. A., Teixeira, L. M., Carvalho Mda, G. \& Beall, B. Sequential multiplex PCR for determining capsular serotypes of pneumococci recovered from Brazilian children. J Med Microbiol 56, 1185-1188 (2007).

15. Tomita, N., Mori, Y., Kanda, H. \& Notomi, T. Loop-mediated isothermal amplification (LAMP) of gene sequences and simple visual detection of products. Nat Protoc 3, 877-882 (2008).

16. Mori, Y. \& Notomi, T. Loop-mediated isothermal amplification (LAMP): a rapid, accurate, and cost-effective diagnostic method for infectious diseases. J Infect Chemother 15, 62-69 (2009).

17. Kim, D. W. et al. Loop-mediated isothermal amplification assay to detect Haemophilus influenzae type b in cerebrospinal fluid. J Clin Microbiol 49, 3621-3626 (2011).

18. Kim, D. W. et al. The enhanced pneumococcal LAMP assay: a clinical tool for the diagnosis of meningitis due to Streptococcus pneumoniae. PLoS One 7, e42954 (2012).

19. Lee, D. et al. Clinical evaluation of a loop-mediated isothermal amplification (LAMP) assay for rapid detection of Neisseria meningitidis in cerebrospinal fluid. PLoS One 10, e0122922 (2015).

20. Torigoe, H., Seki, M., Yamashita, Y., Sugaya, A. \& Maeno, M. Detection of Haemophilus influenzae by loop-mediated isothermal amplification (LAMP) of the outer membrane protein P6 gene. Jpn J Infect Dis 60, 55-58 (2007).

21. Lee, D. et al. A novel loop-mediated isothermal amplification assay for serogroup identification of Neisseria meningitidis in cerebrospinal fluid. Front Microbiol 6, 1548 (2015).

22. Takano, C. et al. Molecular serotype-specific identification of non-type b Haemophilus influenzae by loop-mediated isothermal amplification. Front Microbiol 8, 1877 (2017).

23. Mavroidi, A. et al. Evolutionary genetics of the capsular locus of serogroup 6 pneumococci. J Bacteriol 186, 8181-8192 (2004).

24. Chang, B. et al. Capsule switching and antimicrobial resistance acquired during repeated Streptococcus pneumoniae pneumonia episodes. J Clin Microbiol 53, 3318-3324 (2015).

25. Bentley, S. D. et al. Genetic analysis of the capsular biosynthetic locus from all 90 pneumococcal serotypes. PLoS Genet 2, e31 (2006).

26. Camilli, R. et al. Impact of pneumococcal conjugate vaccine (PCV7 and PCV13) on pneumococcal invasive diseases in Italian children and insight into evolution of pneumococcal population structure. Vaccine 35, 4587-4593 (2017).

27. Ubukata, K. et al. Effects of pneumococcal conjugate vaccine on genotypic penicillin resistance and serotype changes, Japan, 2010-2017. Emerg Infect Dis 24, 2010-2020 (2018).

28. Satzke, C. et al. Pneumococcal carriage in vaccine-eligible children and unvaccinated infants in Lao PDR two years following the introduction of the 13-valent pneumococcal conjugate vaccine. Vaccine 37, 296-305 (2019).

29. Mori, Y. \& Notomi, T. Loop-mediated isothermal amplification (LAMP): Expansion of its practical application as a tool to achieve universal health coverage. J Infect Chemother (2019).

30. Satsangi, J., Jewell, D. P., Welsh, K., Bunce, M. \& Bell, J. I. Effect of heparin on polymerase chain reaction. Lancet 343, 1509-1510 (1994).

31. Al-Soud, W. A., Jonsson, L. J. \& Radstrom, P. Identification and characterization of immunoglobulin G in blood as a major inhibitor of diagnostic PCR. JClin Microbiol 38, 345-350 (2000).

32. Al-Soud, W. A. \& Radstrom, P. Purification and characterization of PCR-inhibitory components in blood cells. J Clin Microbiol 39, 485-493 (2001)

33. Kaneko, H., Kawana, T., Fukushima, E. \& Suzutani, T. Tolerance of loop-mediated isothermal amplification to a culture medium and biological substances. J Biochem Biophys Methods 70, 499-501 (2007).

34. Francois, P. et al. Robustness of a loop-mediated isothermal amplification reaction for diagnostic applications. FEMS immunology and medical microbiology 62,41-48 (2011).

35. Miyamoto, S., Sano, S., Takahashi, K. \& Jikihara, T. Method for colorimetric detection of double-stranded nucleic acid using leuco triphenylmethane dyes. Anal Biochem 473, 28-33 (2015).

36. Anh, D. D. et al. Haemophilus influenzae type B meningitis among children in Hanoi, Vietnam: epidemiologic patterns and estimates of H. Influenzae type B disease burden. Am J Trop Med Hyg 74, 509-515 (2006).

37. Fujitsu, L. PrimerExplorer V5 http://primerexplorer.jp/e/ (2016).

38. Mori, Y., Kitao, M., Tomita, N. \& Notomi, T. Real-time turbidimetry of LAMP reaction for quantifying template DNA. J Biochem Biophys Methods 59, 145-157 (2004).

39. Kennedy, W. A. et al. Incidence of bacterial meningitis in Asia using enhanced CSF testing: polymerase chain reaction, latex agglutination and culture. Epidemiol Infect 135, 1217-1226 (2007).

\section{Acknowledgements}

M. Seki has been supported by JSPS Bilateral Open Partnership Joint Research Projects. DWK was supported by the grant NRF-2018R1A2A2A05018341 and NRF-2015M3C9A2054024 from National Research Foundation (NRF) of Korea.

\section{Author contributions}

P.K., D.K., M. Seki, S.H. contributed the conception of this study; D.K., M. Seki designed the experiments; C.T., Y.K., K.F., D.O., M.S., D.K., M. Seki performed the experiments; B.C., M.O., E.K., P.K. acquired samples; C.T., D.K., M. Seki, T.H. analyzed data; C.T., S.H., P.K., B.C., M.O., E.K., D.K., M. Seki, T.H. interpreted data; C.T., Y.K., P.K., S.H., D.K., M. Seki drafted the manuscript; and K.F., D.O., B.C., M.O., E.K., M.S., T.H. approved the manuscript. 


\section{Competing interests}

The authors declare no competing interests.

\section{Additional information}

Supplementary information is available for this paper at https://doi.org/10.1038/s41598-019-56225-0.

Correspondence and requests for materials should be addressed to M.S. or D.W.K.

Reprints and permissions information is available at www.nature.com/reprints.

Publisher's note Springer Nature remains neutral with regard to jurisdictional claims in published maps and institutional affiliations.

(c) (i) Open Access This article is licensed under a Creative Commons Attribution 4.0 International License, which permits use, sharing, adaptation, distribution and reproduction in any medium or format, as long as you give appropriate credit to the original author(s) and the source, provide a link to the Creative Commons license, and indicate if changes were made. The images or other third party material in this article are included in the article's Creative Commons license, unless indicated otherwise in a credit line to the material. If material is not included in the article's Creative Commons license and your intended use is not permitted by statutory regulation or exceeds the permitted use, you will need to obtain permission directly from the copyright holder. To view a copy of this license, visit http://creativecommons.org/licenses/by/4.0/.

(C) The Author(s) 2019 